\title{
Improving extraction efficiency of third integer resonant extraction using higher order mode multipoles
}

\author{
K.A. Brown, V. Schoefer, M. Tomizawa
}

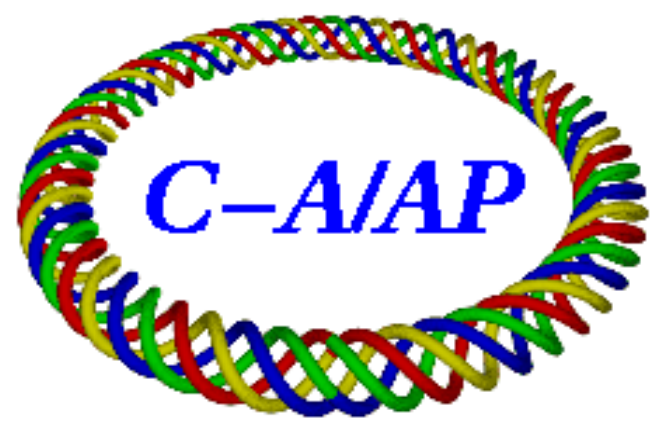

Collider-AcceleratorDepartment Brookhaven National Laboratory

Upton, NY 11973

\section{U.S. Department of Energy}

Office of Science, Office of Nuclear Physics

Notice: This document has been authorized by employees of Brookhaven Science Associates, LLC under Contract No. DE-SC0012704 with the U.S. Department of Energy. The United States Government retains a nonexclusive, paid-up, irrevocable, world-wide license to publish or reproduce the published form of this document, or allow others to do so, for United States Government purposes. 


\title{
Improving Extraction Efficiency of Third Integer Resonant Extraction using Higher Order Multipoles
}

\author{
K.A.Brown* and V. Schoefer \\ C-AD Dept., BNL, Upton, NY 11973-5000 \\ M. Tomizawa ${ }^{\dagger}$ \\ KEK, High Energy Accelerator Research Organization, \\ Oho 1-1, Tsukuba-Shi, Ibaraki 305-0801, Japan
}

\begin{abstract}
The new accelerator complex at J-PARC will operate with both high energy and very high intensity proton beams [1]. With a design slow extraction efficiency of greater than $99 \%$ this facility will still be depositing significant beam power onto accelerator components [2]. To achieve even higher efficiencies requires some new ideas. The design of the extraction system and the accelerator lattice structure leaves little room for improvement using conventional techniques. In this report we will present one method for improving the slow extraction efficiency at J-PARC by adding duodecapoles or octupoles to the slow extraction system. We will review the theory of resonant extraction, describe simulation methods, and present the results of detailed simulations. From our investigations we find that we can improve extraction efficiency and thereby reduce the level of residual activation in the accelerator components and surrounding shielding.
\end{abstract}

PACS numbers: 41.85.-p, 41.85.Ar, 29.27.-a, 29.27.Ac, 29.27.Bd 


\section{INTRODUCTION}

The method of extracting particles from accelerators using transverse resonances was first proposed by J.L. Tuck and L.C. Teng [3] in 1951. The method was first demonstrated to work at the Liverpool cyclotron in 1953 [4] by A.V. Crewe. The first detailed quantitative theoretical treatment was done by K.J. Le Couteur $[5,6]$. The application to the CERN PS was proposed by H.G. Hereward in 1961 [7, 8]. Although Hereward considered multiple resonance conditions, he focused mostly on extraction near the integer or half-integer, using sextupoles for non-linear growth. The application to the BNL AGS was proposed by M.Q. Barton in 1966 [9]. For the AGS, extraction was done on the 2/3 integer. The first modern theoretical treatment for strong focusing synchrotrons was done by Kobayashi and Takahashi [10], which was built on Herewards work and the experimental results by Bovet, Lambertson, and Reich [11]. The literature on resonant extraction is rich and covers half a century of journal reports and conference proceedings, as well as a great number of internal reports. A full historical treatment of the subject is well beyond the scope of this report. Of interest to this report, though, is the literature shows that explorations of higher order multipoles to give the resonances strong tune amplitude dependence were made very early in the development of this technique. Hereward, Le Couteur, and Kobahashi all explored different combinations of multipoles on integer, half-integer, and third integer resonances. In more recent history the study of higher order multipoles and resonances has provided deep insights into these chaotic regimes $[12,13]$. Finally, the concept of increasing the step size while keeping the beam size confined in the extraction acceptance channel through the use of higher order multipoles has been discussed among ourselves and appears first, as far as we can determine in a literature search, in a 1997 conference report by N.N. Alexeev, S.L. Bereznitsky, and A.E. Bolshakov [14].

The purpose of resonant extraction is to allow particles to be removed from the accelerator very slowly and transported to fixed targets. Historically, slow extraction has been very useful for experiments since it gave a steady stream of events at low rates. It also reduced the instantaneous power delivered onto the targets, which might otherwise be damaged. As detector and fixed target technologies improved and rates could be increased, the intensity of the slow extracted beams was increased. Of course, this means the efficiencies of the process needed to be improved. Today it is well established that extraction efficiencies on 
the order of $98 \%$ can be achieved with very intense proton beams [15]. High efficiencies are also required for high energy extraction, which has been demonstrated at FNAL [16] and at CERN [17, 18].

In this report we will briefly review the formalism and ideas behind resonant extraction. We will expand upon the traditional treatment by adding higher order multiples. We will then present a method for improving the extraction system at J-PARC. The method we will discuss will describe the use of higher order mulitpoles in the lattice structure. This will allow the step size of the extracted particles to be increase without increasing the acceptance of the extraction channel. By employing these methods we will show that the extraction efficiency can be increased, reducing activation of accelerator components.

\section{SEXTUPOLES AND HIGHER ORDER MULTIPOLES}

A thin sextupole of length $L$, pole tip field of $B_{r}$, and radius of bore $r$, changes the trajectory of a particle, with a rigidity $B \rho$, as follows.

$$
\begin{gathered}
\Delta x=0 \\
\Delta x^{\prime}=\frac{1}{2} \frac{L}{B \rho}\left(\frac{\partial^{2} B_{r}}{\partial r^{2}}\right)_{0}\left(x^{2}-y^{2}\right)
\end{gathered}
$$

Transforming into normalized phase space coordinates using,

$$
\Delta x^{\prime}=\frac{1}{\sqrt{\beta_{x}}} \Delta X^{\prime}, \quad x=\sqrt{\beta_{x}} X, \quad y=\sqrt{\beta_{y}} Y
$$

where we have made use of the Courant-Snyder lattice parameter, $\beta_{x}$, (1) becomes

$$
\begin{gathered}
\Delta X=0 \\
\Delta X^{\prime}=S\left(X^{2}-\frac{\beta_{y}}{\beta_{x}} Y^{2}\right)
\end{gathered}
$$

where,

$$
S=\frac{\beta_{x}^{3 / 2}}{2} \frac{L}{B \rho}\left(\frac{\partial^{2} B_{r}}{\partial r^{2}}\right)_{0}
$$


In the vertical plane,

$$
\begin{gathered}
\Delta Y=0 \\
\Delta Y^{\prime}=-2 S \frac{\beta_{y}}{\beta_{x}} X Y
\end{gathered}
$$

A thin duodecapole changes the trajectory of particles passing through it as follows.

$$
\begin{gathered}
\Delta x=0 \\
\Delta x^{\prime}=\frac{1}{120} \frac{L}{B \rho}\left(\frac{\partial^{5} B_{r}}{\partial r^{5}}\right)_{0}\left(x^{5}+5 x y^{4}-10 x^{3} y^{2}\right)
\end{gathered}
$$

Transforming into normalized phase space coordinates, (6) becomes

$$
\begin{gathered}
\Delta X=0 \\
\Delta X^{\prime}=\mathcal{D}\left(X^{5}+5 X\left(\frac{\beta_{y}}{\beta_{x}}\right)^{2} Y^{4}-10 \frac{\beta_{y}}{\beta_{x}} X^{3} Y^{2}\right)
\end{gathered}
$$

where,

$$
\mathcal{D}=\frac{\beta_{x}^{3}}{120} \frac{L}{B \rho}\left(\frac{\partial^{5} B_{r}}{\partial r^{5}}\right)_{0}
$$

In the vertical plane,

$$
\begin{gathered}
\Delta Y=0 \\
\Delta Y^{\prime}=-\mathcal{D}\left(\frac{\beta_{y}}{\beta_{x}}\right)^{3}\left(Y^{5}+5 Y\left(\frac{\beta_{x}}{\beta_{y}}\right)^{2} X^{4}-10 \frac{\beta_{x}}{\beta_{y}} Y^{3} X^{2}\right)
\end{gathered}
$$

\section{HAMILTONIAN FORMALISM}

The mathematical formalism for describing particle motion during resonant extraction is very well described in ref. [19] and the formalism for what is now called the Kobayashi Hamiltonian is found in ref. [10]. In this section we will only provide a brief review of the 
Hamiltonian dynamics of slow extraction. In the case with a sextupole only, the three turn Hamiltonian (Kobayashi Hamiltonian) is

$$
H=\frac{\epsilon}{2}\left(X^{2}+X^{\prime 2}\right)+\frac{S}{4}\left(3 X X^{\prime 2}-X^{3}\right)
$$

where for every three turns

$$
\begin{gathered}
\Delta X_{3}=\epsilon X_{0}^{\prime}+\frac{3}{2} S X_{0} X_{0}^{\prime} \\
\Delta X_{3}^{\prime}=-\epsilon X_{0}+\frac{3}{4} S\left(X_{0}^{2}-X_{0}^{\prime 2}\right) .
\end{gathered}
$$

$\epsilon$ represents a small phase shift, where

$$
\epsilon=6 \pi \delta Q=6 \pi\left(Q_{\text {particle }}-Q_{\text {resonance }}\right) .
$$

Here $Q_{\text {particle }}$ is the single particle tune and $Q_{\text {resonance }}$ is the central tune of the resonance. When

$$
H=\left(\frac{2 \epsilon}{3}\right)^{3} / S^{2}
$$

the Hamiltonian can be factored into three terms, each of which describes a straight line in normalized phase space.

$$
\left(\frac{S}{4} X+\frac{\epsilon}{6}\right)\left(\sqrt{(3)} X^{\prime}+X-\frac{4 \epsilon}{3 S}\right)\left(\sqrt{3} X^{\prime}-X+\frac{4 \epsilon}{3 S}\right)=0
$$

The area of the stable region contained within the triangle described by these three lines is defined by the ratio of $\epsilon / S$.

$$
A=3 \sqrt{3}\left(\frac{2}{3} \frac{\epsilon}{S}\right)^{2}
$$

From this it is clear there is a tune amplitude dependence in the resonance that is a function of the strength of the sextupoles and a particles betatron amplitude. This is important because the methods used to drive particles into the resonance will depend on either changing the betatron tune or the betatron amplitude, or perhaps even both.

We call the area contained within a single particles unperturbed motion the single particle emittance, written as 


$$
E=a^{2} \pi \text { where, } a^{2}=X^{2}+X^{\prime 2}
$$

The stable area of motion is then,

$$
E_{\text {stable }} \leq \frac{48 \sqrt{3} \pi}{S^{2}}\left(\delta Q^{2}\right) \pi
$$

In the case in which we include a duodecapole, the three turn Hamiltonian is,

$$
H=\frac{\epsilon}{2}\left(X^{2}+X^{\prime 2}\right)+\frac{S}{4}\left(3 X X^{\prime 2}-X^{3}\right)+\frac{1}{64} \mathcal{D}\left(11 X^{6}+15 X^{4} X^{\prime 2}+45 X^{2} X^{\prime 4}+9 X^{\prime 6}\right)
$$

where for every three turns

$$
\begin{gathered}
\Delta X_{3}=\epsilon X_{0}^{\prime}+\frac{3}{2} S X_{0} X_{0}^{\prime}-\frac{3}{32} \mathcal{D}\left(5 X_{0}^{4} X_{0}^{\prime}+30 X_{0}^{2}{X_{0}^{\prime}}^{3}+9 X_{0}^{\prime 5}\right) \\
\Delta X_{3}^{\prime}=-\epsilon X_{0}+\frac{3}{4} S\left(X_{0}{ }^{2}-X_{0}^{\prime 2}\right)+\frac{1}{32} \mathcal{D}\left(33 X_{0}{ }^{5}+30 X_{0}^{3}{X_{0}^{\prime}}^{2}+45 X_{0} X_{0}^{\prime 4}\right)
\end{gathered}
$$

Transforming into action-angle variables using

$$
\begin{aligned}
& X=\sqrt{2 J} \cos \theta \\
& X^{\prime}=\sqrt{2 J} \sin \theta
\end{aligned}
$$

then,

$$
\mathcal{H}=-\left(\frac{J^{3} \mathcal{D}(10+\cos (6 \theta))}{8}\right)-\frac{J^{3 / 2} S \cos (3 \theta)}{\sqrt{2}}+J \epsilon
$$

In this form it is easy to see that a sextupole perturbation will create three unstable fixed points in phase space, with the distortion of the Hamiltonian surface being a function of the sextupole strength and the particle amplitude. A duodecapole creates two perturbative terms. One term, as with the sextupole, creates six unstable fixed points in phase space, 
with the distortion of the surface being a function of the duodecapole strength and the particle amplitude. The second term is independent of azimuth, but is a function of the duodecapole strength and the particle amplitude. None of the perturbative terms depend on the particle betatron tune.

To visualize Eq. 21, the motion of particles over many revolutions and with different amplitudes can be mapped, showing the Hamiltonian surface of sections, as shown in figure 1, in which the duodecapole term has been set to 0. Figure 2 shows the Hamiltonian surface for the same parameters. Turning on the duodecapole term, using realistic values, distorts the Hamiltonian surface, as shown in figure 3. Note that the parameter values used in these figures were chosen purely for illustrative purposes.

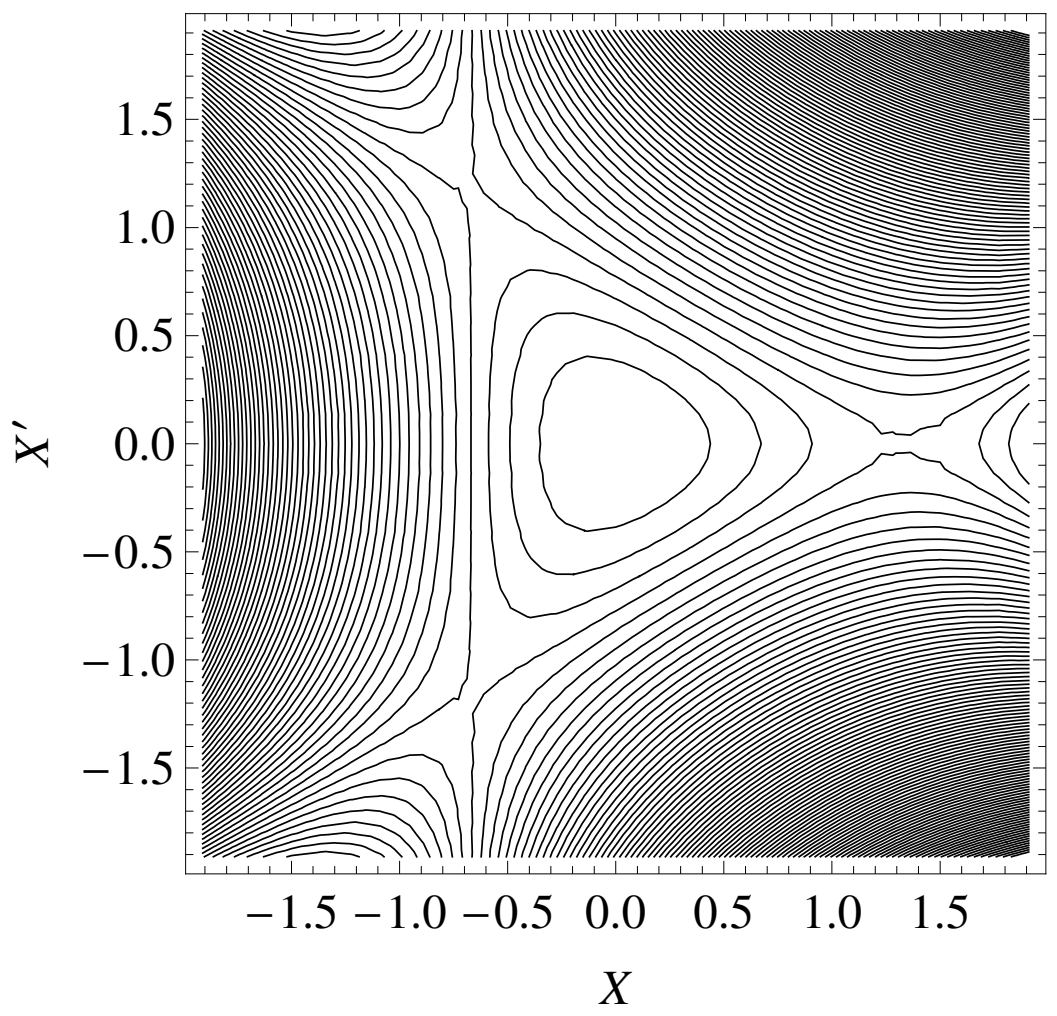

FIG. 1: Hamiltonian surface of section for Eqs. 10 and 19 with $\epsilon=0.001$ and $S=0.0005$

\section{J-PARC SLOW EXTRACTION}

The J-PARC slow extraction system is a unique design, with a lattice that has a negative $\gamma_{t r}$ and both horizontal and vertical chromaticities defined to be zero (or very near zero). 


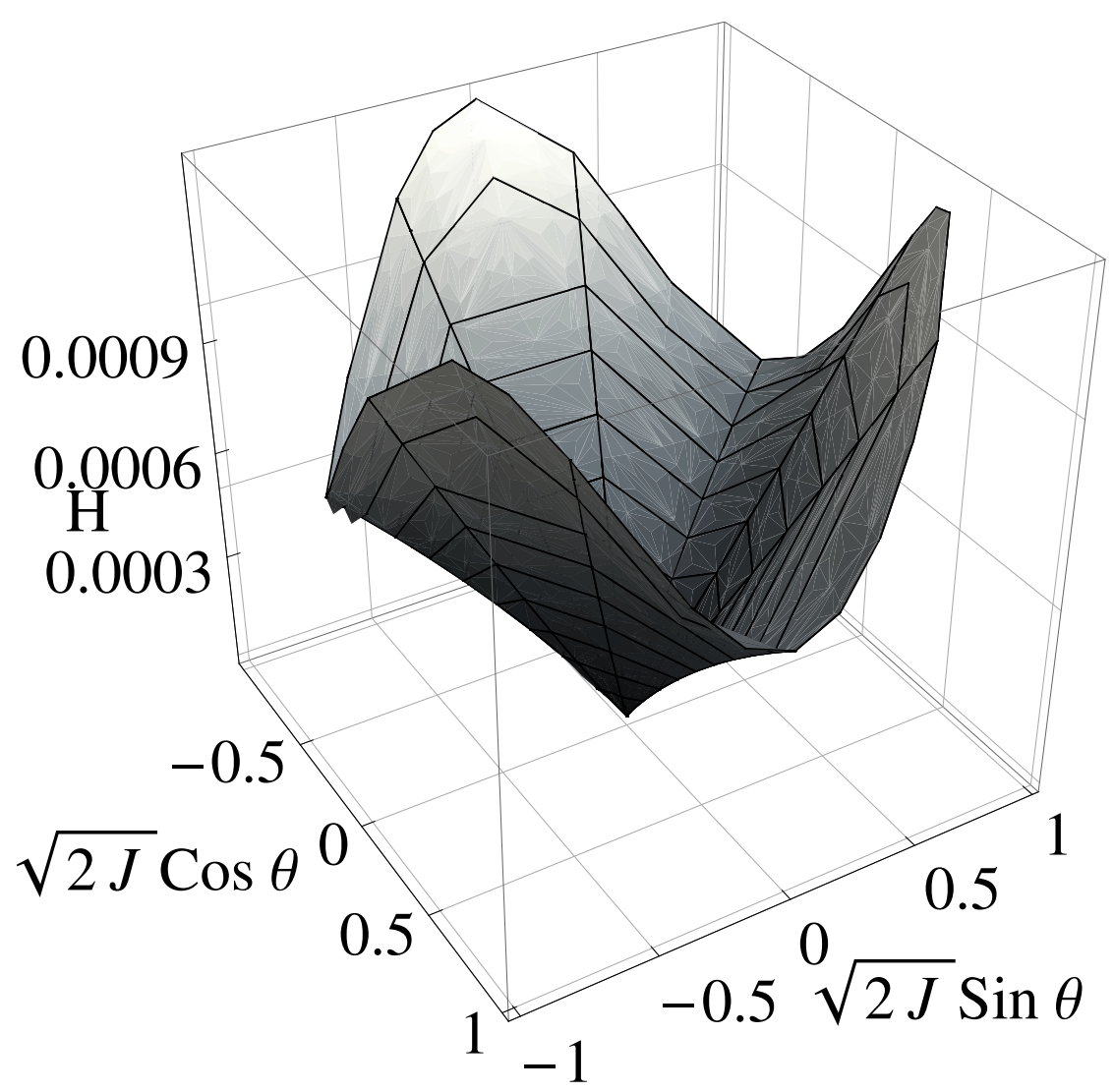

FIG. 2: 3D Hamiltonian surface for Eq. 21 with $\epsilon=0.001, S=0.0005$, and $\mathcal{D}=0.0$

The dispersion at the electrostatic septum is defined to be zero and the electrostatic septa are located to the inside of the accelerator aperture. The horizontal betatron tune is set to be below the tune of the resonance, requiring the horizontal tune to be ramped more positive during the extraction process. In combination with the near zero chromaticity this has the effect of extracting large amplitude particles first and then slowly peeling the beam in phase space until small amplitude particles are extracted last. In order to minimize the extracted beam emittance a dynamic bump is employed, which keeps the angular distribution of the extracted particles minimized. This will also improve the extraction efficiency, in that losses on downstream septa are minimized due to the rotation of the extracted phase space and the alignment of the septa.

The nominal design for the J-PARC slow extraction uses a dynamic bump that minimizes the angular spread of the extracted beam, but does not maintain a constant step size at the septum. The range in positions and angle for this bump, for 3 different particle amplitudes $(0,2$, and $6 \pi \mathrm{mm}$-mrad (un-normalized)) is shown in figure 4 . The extraction system 


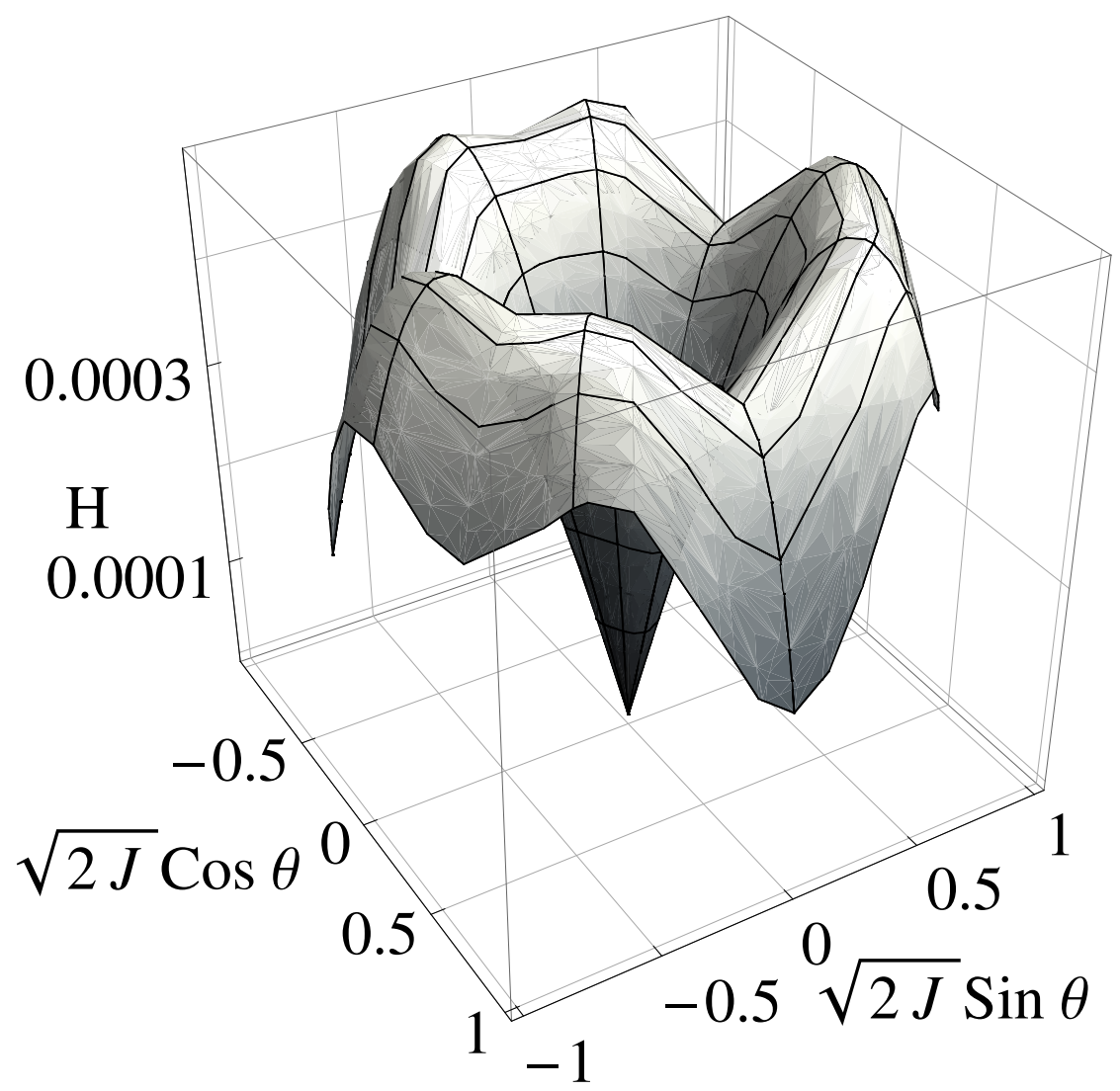

FIG. 3: 3D Hamiltonian surface for Eq. 21 with $\epsilon=0.001, S=0.0001$, and $\mathcal{D}=0.00055$

consists of two electrostatic septa (ESS1 and ESS2), located on the inside of the accelerator aperture, followed by 10 magnetic septa (SM11 through SM33).

Figure 5 shows the phase space at the entrance to the first electrostatic septum (ESS1). The extraction efficiency is $99 \%$. This efficiency is the minimum extraction efficiency, since the simulation assumes that any particle that "hits" the ESS1 is lost.

\section{IMPROVING EXTRACTION EFFICIENCY}

Extraction efficiency is normally defined as the ratio of the number of particles that get extracted from the accelerator successfully to the number that are circulating in the accelerator prior to extraction. If we assume that only particles that hit the septum are not extracted, then the extraction efficiency is also equal to the ratio of the number of particles that successfully step across the septum into the deflecting field of the septum to the number circulating prior to extraction. The probability of a particle hitting the septum is a function 


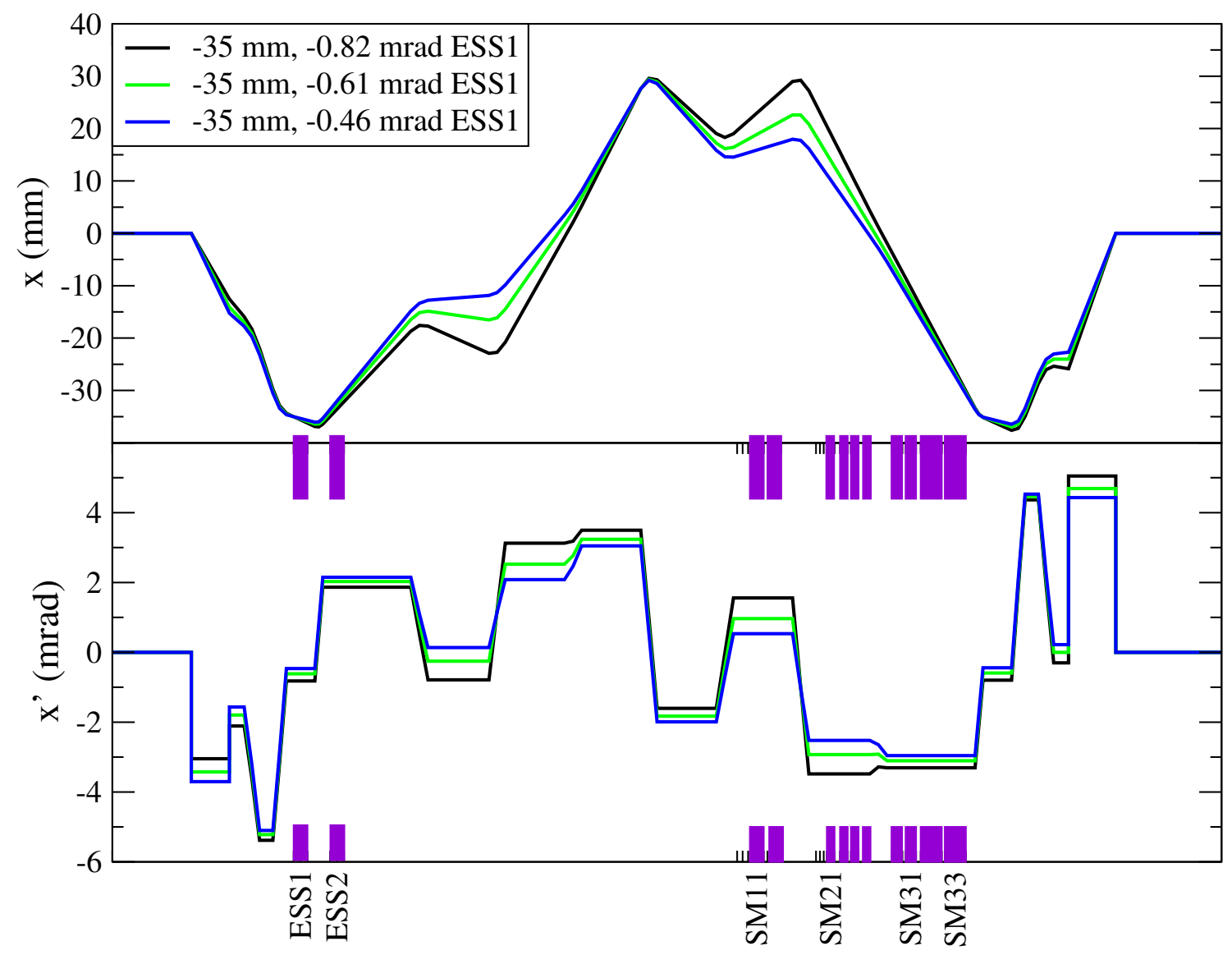

FIG. 4: Change in orbit position and angle in extraction region for dynamic bump with constant position at ESS1 and angle at ESS1 that varies from 0 to $-0.25 \mathrm{mrad}$

of the size of the step that the particle makes between its arrival just past the septum to just before (3 turns prior). This step is usually called the step size. As the trajectory of a particle takes it from the unstable fixed points, where it first is excited into non-linear growth from the resonance, to where it crosses the septum, it is said to spiral out from its original stable trajectory. As the particle spirals out, the steps increase in size non-linearly (by the square of the distance from the unstable fixed point). Particles that spiral out very quickly (in very few turns) will have a smaller step size at the septum than particles that had more time to form a larger step size, roughly speaking. The number of turns it takes for a particle to spiral out and be extracted is a function of its initial amplitude in phase space. So it is possible to define the extraction efficiency in terms of step size. This is easily measured in particle tracking simulations. The extraction efficiency is then simply one minus the ratio of the septum thickness to the average step size. So for a tracking simulation to determine 


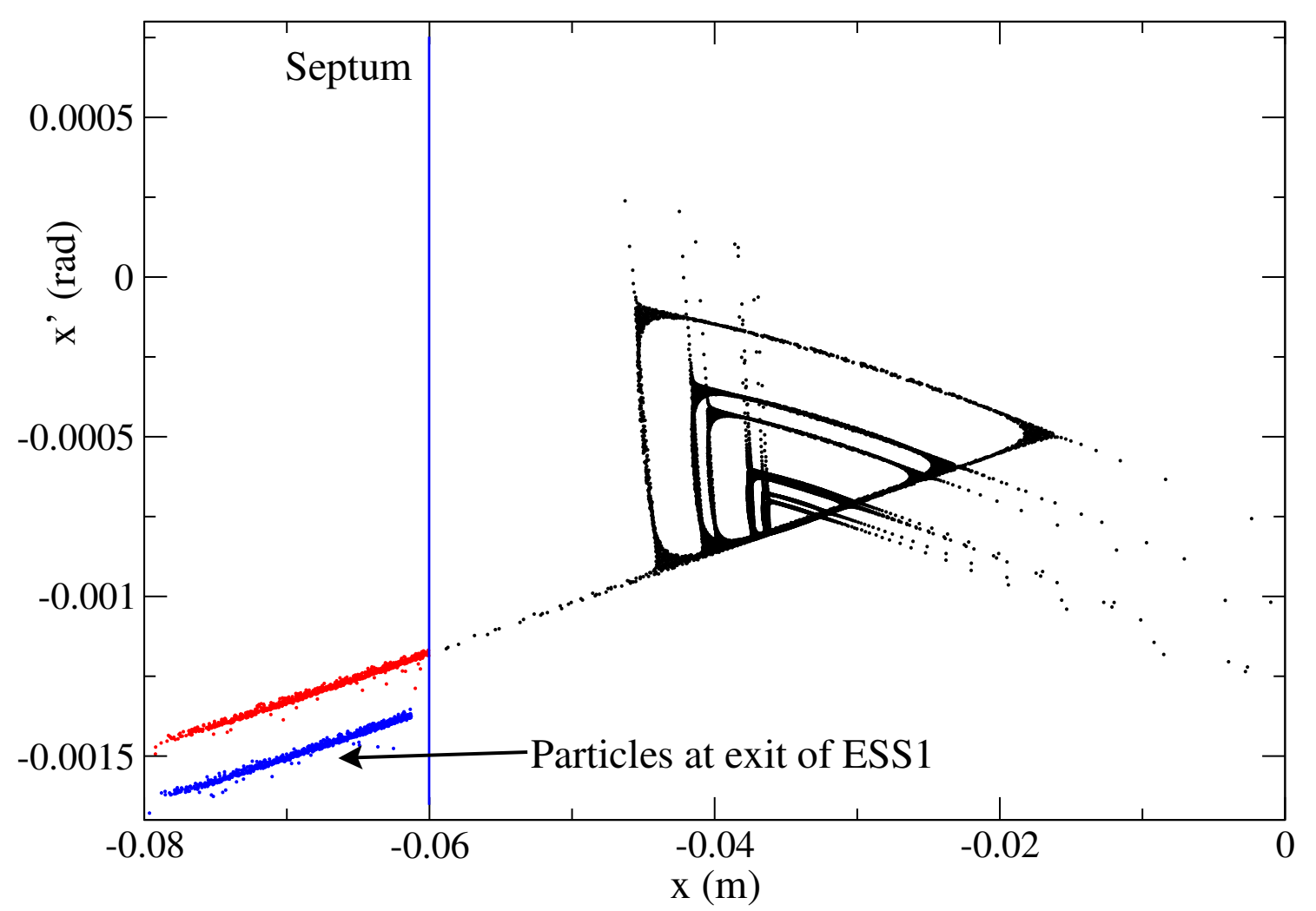

FIG. 5: Phase space at J-PARC ESS for 10 different particles (in black), including a dynamic bump. Step size in this example is $2 \mathrm{~cm}$ for small amplitude particles. $>990$ Particles in red have stepped past the septum (blue line at $-0.06 \mathrm{~m}$ ) out of 1000 initial particles. In this case the initial beam emittance is $2 \pi \mathrm{mm}$-mrad. The septum thickness is taken to be $100 \mu \mathrm{m}$. Extracted particle phase space trajectories are also shown at the exit of ESS1.

efficiency, it just has to keep track of the step size distributions of the particles that are being extracted.

The particle tracking simulations follow the motion of charged particles in linear systems of magnets that can be described as a simple harmonic oscillator with the form: (Most of what appears here is found described in much greater detail in [22], although we keep to the notation we have been using throughout this report.)

$$
\frac{d^{2} X}{d \phi^{2}}+\nu^{2} X=0
$$

where $\nu$ is the betatron tune and $\phi$ is the phase at a given point in the system. The general solution to this equation is, 


$$
X(\phi)=A \cos (\nu \phi+\delta)
$$

where $\mathrm{A}$ and $\delta$ are constants of integration which depend on the initial conditions. As is standard practice in these kinds of simulations we use the canonical transformations between $\left(X, X^{\prime}\right)$ coordinates and $\left(x, x^{\prime}\right)$ coordinates (Floquet transformation),

$$
\begin{gathered}
X=\frac{x}{\sqrt{\beta}} \\
X^{\prime}=\frac{x \alpha}{\sqrt{\beta}}+x^{\prime} \sqrt{\beta}
\end{gathered}
$$

To do tracking simulations of single particles through arbitrary linear systems we generate a distribution of coordinates using random numbers, generating an initial Gaussian distribution. A simplified lattice is described in which basic elements are defined. Then non-linear elements are added, as thin "kicks". All transformations are kept symplectic, since we are tracking particles over many revolutions. For a sextupole "kick" we use eq. 3 and for a duodecapole we use eq. 7 .

Figure 6 shows slow extraction for three different cases (although for unrealistic conditions that allow demonstrating the affect of the duodecapoles clearly). Case 1 is for normal slow extraction using just sextupoles, with strengths set to give about a $12 \mathrm{~cm}$ maximum step size. There is slight curvature to the particle trajectories, as expected, since the simulation is based on the equation of motion, eq. 22, not the simplified Hamiltonians. In all three of the following figures $(6,7$, and 8 ), the black dots or lines are for case 1 , the red dots or lines are for case 2, and the blue dots or lines are for case 3. For Case 2 the duodecapoles are excited with polarities such that the particle flows are forced clockwise in the Hamiltonian surface of section, causing the phase space of the extracted particles to have an upward curvature, as seen in figure 7. For Case 3 the duodecapoles are excited with polarities that drive the particle flows counter-clockwise. Figure 8 shows the step size distributions for the three cases. As can clearly be seen, for Case 2 the extraction efficiency is worse than the normal case (average step size is smaller) while for Case 3 the extraction efficiency is improved. Scaling these values down to a more realistic case, given that the extraction efficiency for Case 1 is $99 \%$, would imply that for Case 2 the efficiency is significantly less than $99 \%$ while for Case 3 the efficiency is improved to about $99.3 \%$. To see how this might work using 


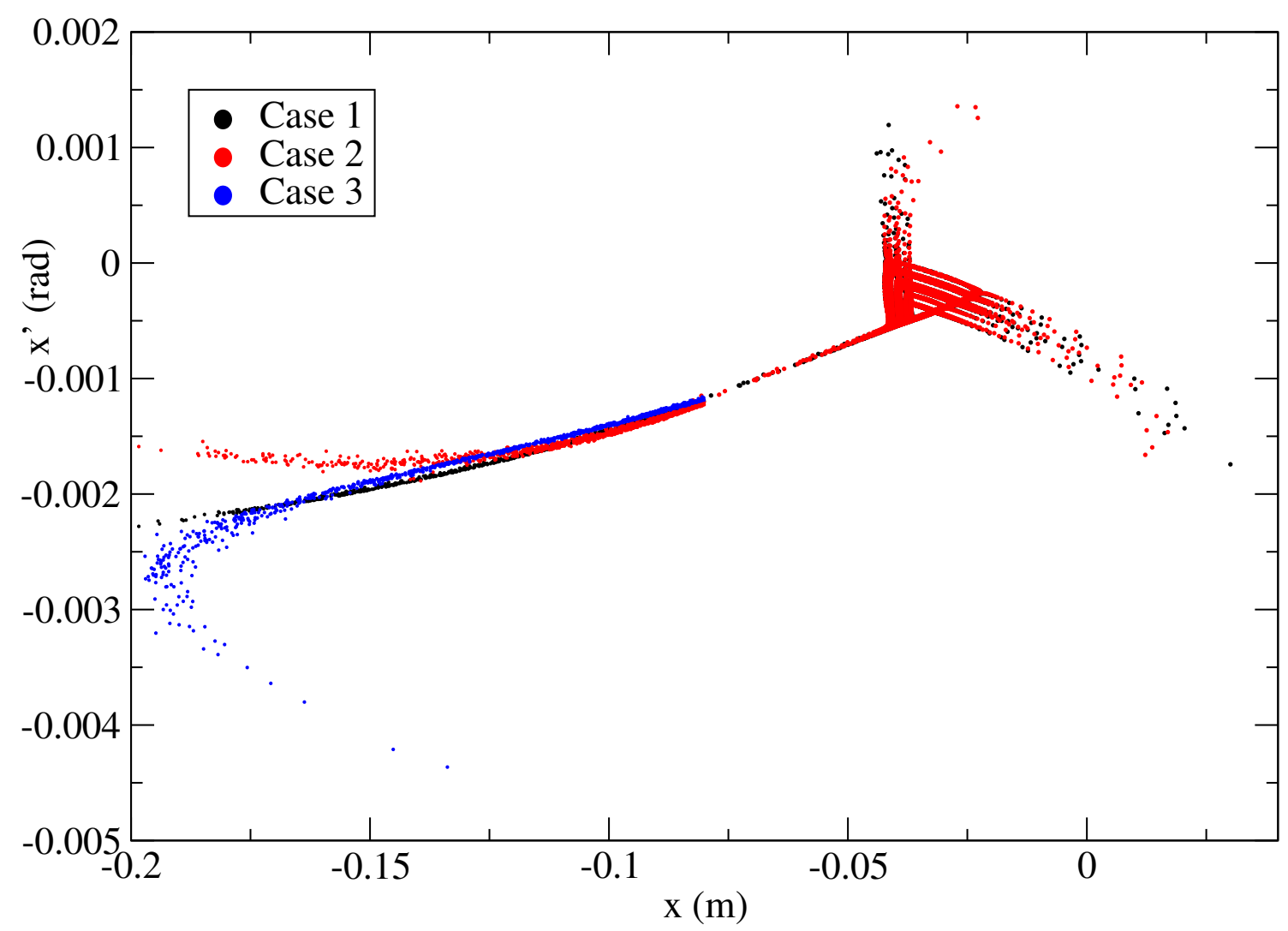

FIG. 6: Extracted beam phase space and seperatrix for slow extraction with and without the use of duodecapoles. This shows an exaggerated case with conditions set to create a large step size, to show the effect of the duodecapoles. The black points are for normal slow extraction without the duodecapoles.

more realistic conditions we repeated the simulation of figure 5 , this time with and without the duodecapoles, and with and without octupoles. Figure 9 shows the result of simulating 10000 particles. Figure 10 shows the step size distributions from these simulations. In the simulation the higher order multipoles were located at the same location in the lattice as the drive sextupoles, with the same polarities (making for a total of 8 drive sextupoles and 8 higher order multipoles in the lattice). The strength of the duodecapoles and the octupoles were defined to be about that of a $1 \mathrm{~T}$ pole tip field in a $20 \mathrm{~cm}$ aperture, $1 \mathrm{~m}$ long magnet, with the extraction momentum at $50 \mathrm{GeV} / \mathrm{c}$. The improvement in the extraction efficiency is not as good as in the unrealistic case, but the effect is significant enough to reduce the deposited beam power on the accelerator components. Using the lower order octupoles instead of the duodecapoles enhances the affect slightly, since an octupole will have more 
field near the center of the magnet than a duodecapole, given the same pole tip field and magnet bore. This demonstrates that what is important is for the larger amplitude particles to be bent back inward, confining them within the aperature of the extraction septum, and so any higher order multiple, than the sextupoles, will be effective.

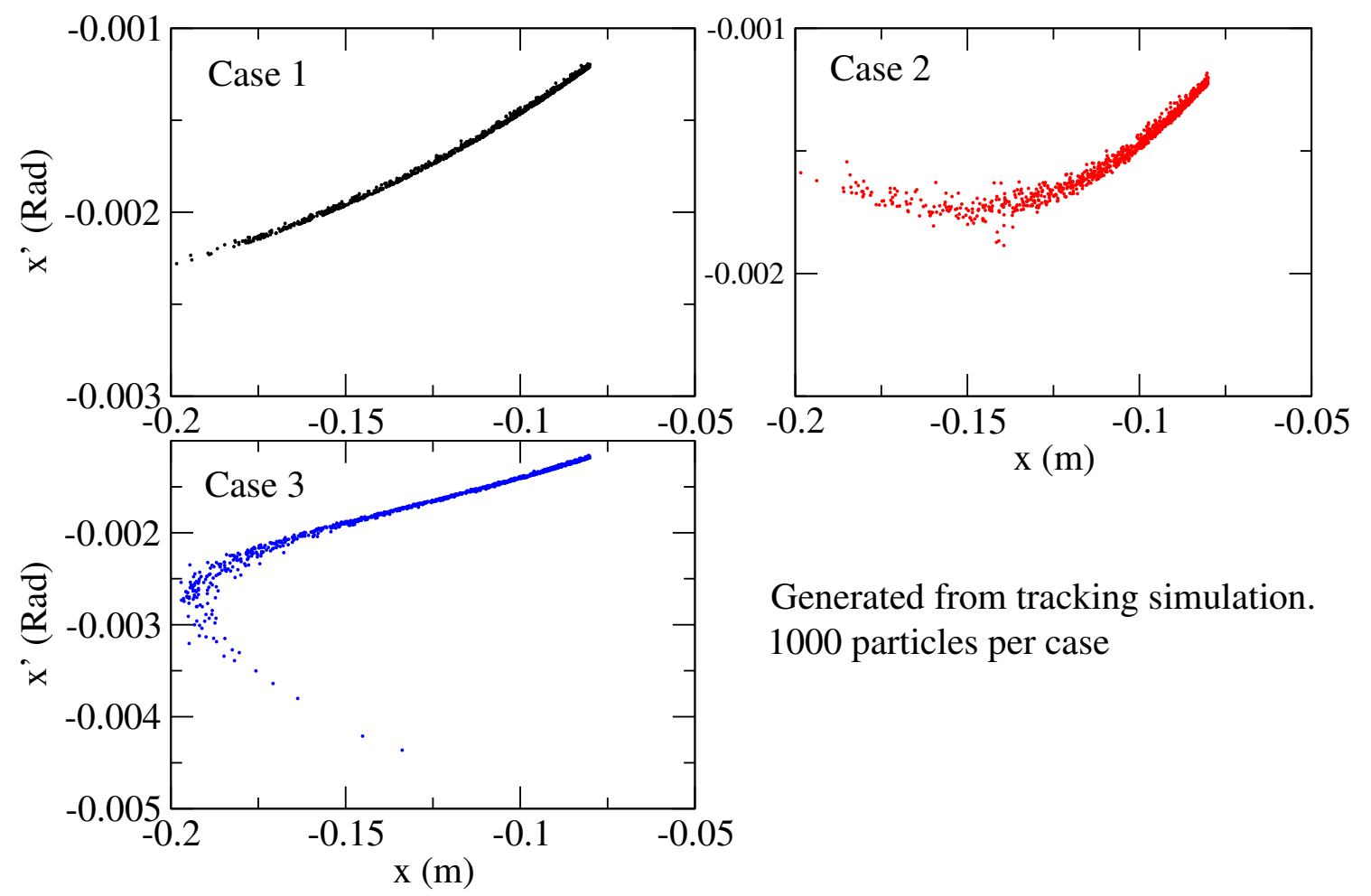

FIG. 7: Phase space distributions for extracted particles for 3 cases discussed in text. 

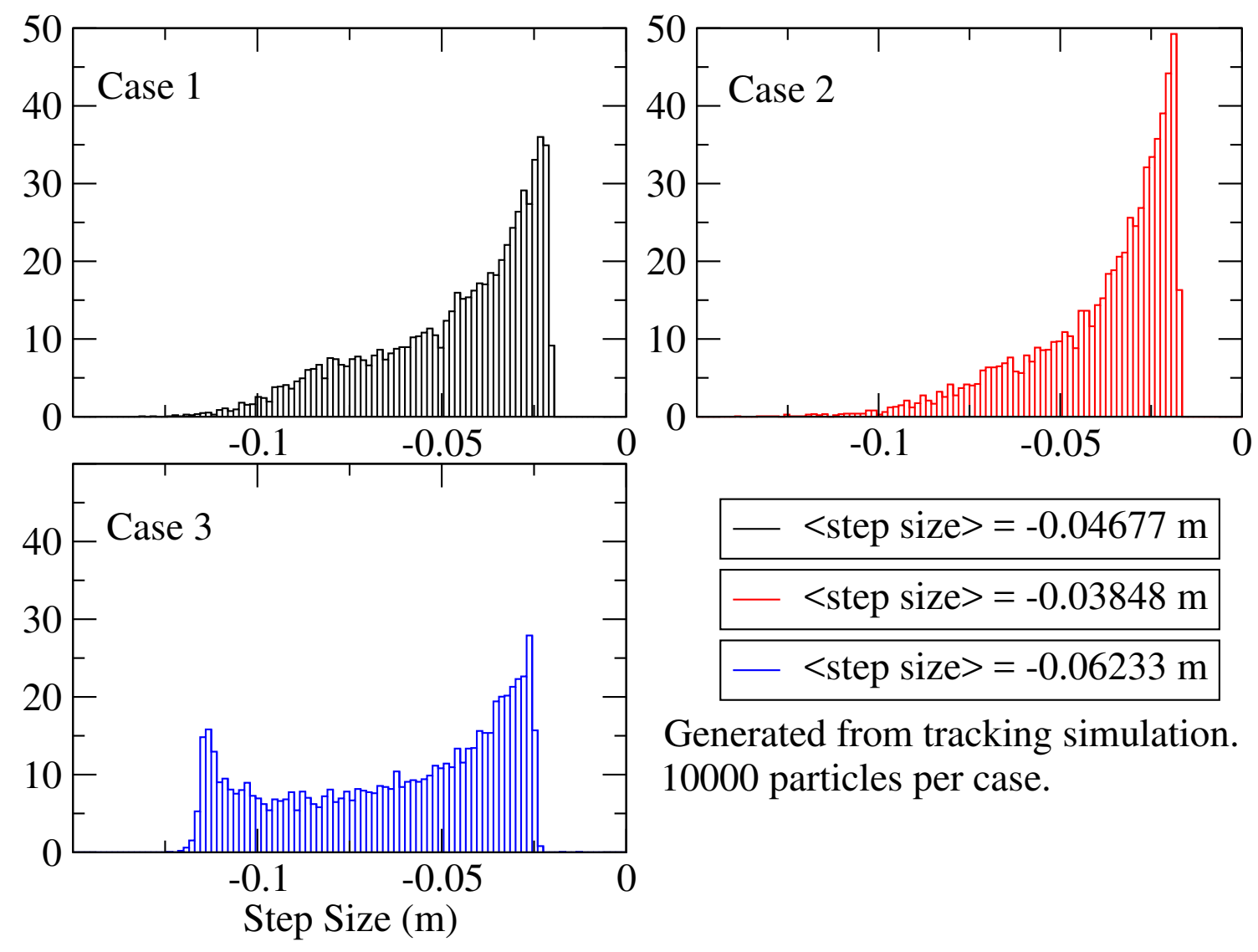

Generated from tracking simulation. 10000 particles per case.

FIG. 8: Step size distributions for the 3 cases discussed in the text. The average step sizes are given for each case.

The actual amount of beam power deposited on accelerator components is less than would be estimated if we assumed that all particles that hit the septum are lost. In the case of the J-PARC design, a large fraction of the particles that hit the septum are scattered slightly and have a slightly reduced momentum, but still are able to be extracted through the extraction channel into the transport line. Although they don't contribute to activation in the accelerator components, these particles do form a momentum tail on the particle distribution and form a halo due to the larger angular spread introduced by the scattering. The higher order multipoles reduce the number of particles that hit the septum from about $1 \%$ to about $0.88 \%$. The beam power that contributes to activation of the extraction devices is estimated to be about 1.5 to $2 \mathrm{~kW}$. This could be reduced to by a few hundred Watts with the addition of the higher order multipoles. In addition the amount of halo and momentum tail produced will be reduced by the same factor. 


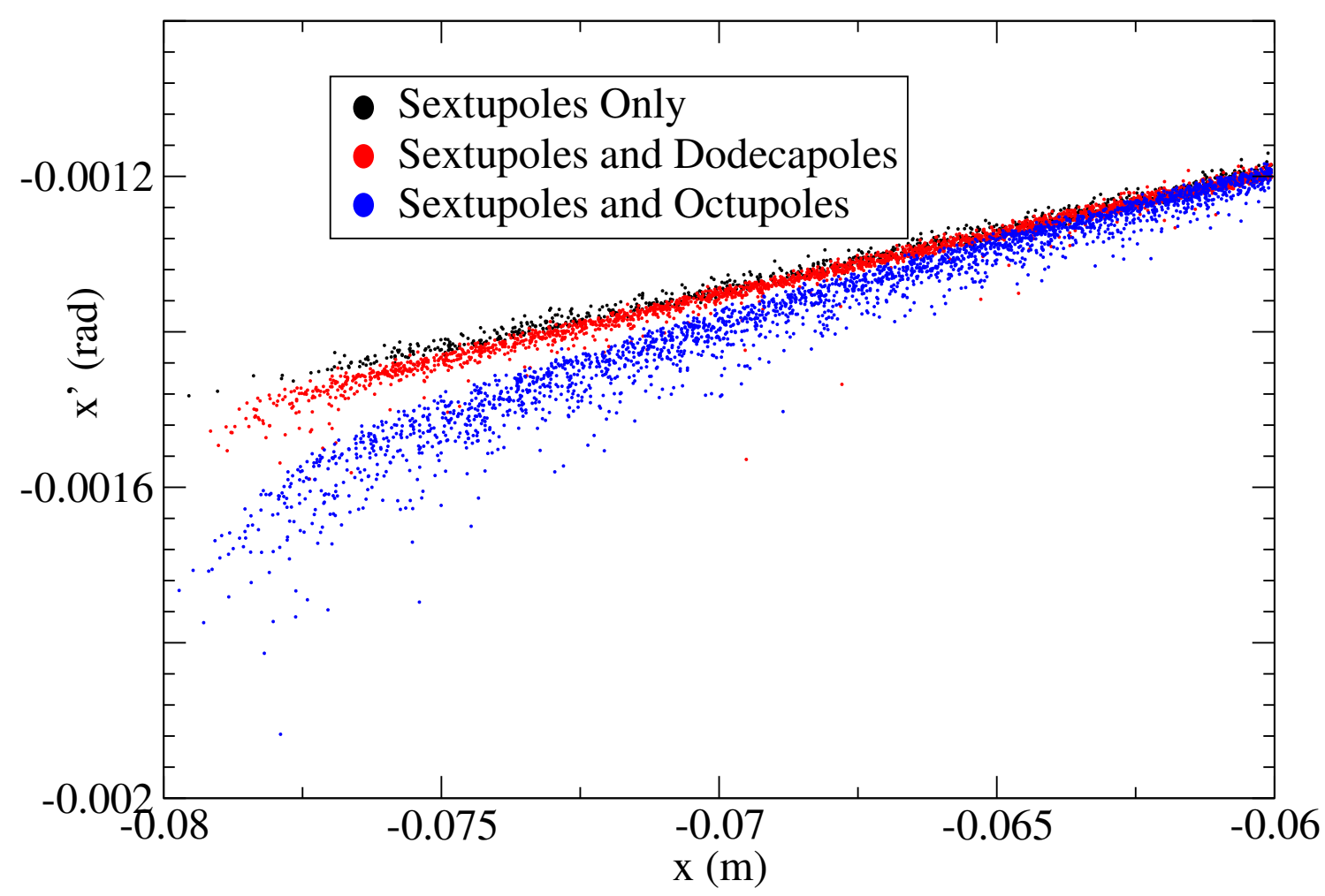

FIG. 9: Phase space distributions for extracted particles for normal J-PARC slow extraction, with and without the higher order multipoles. 


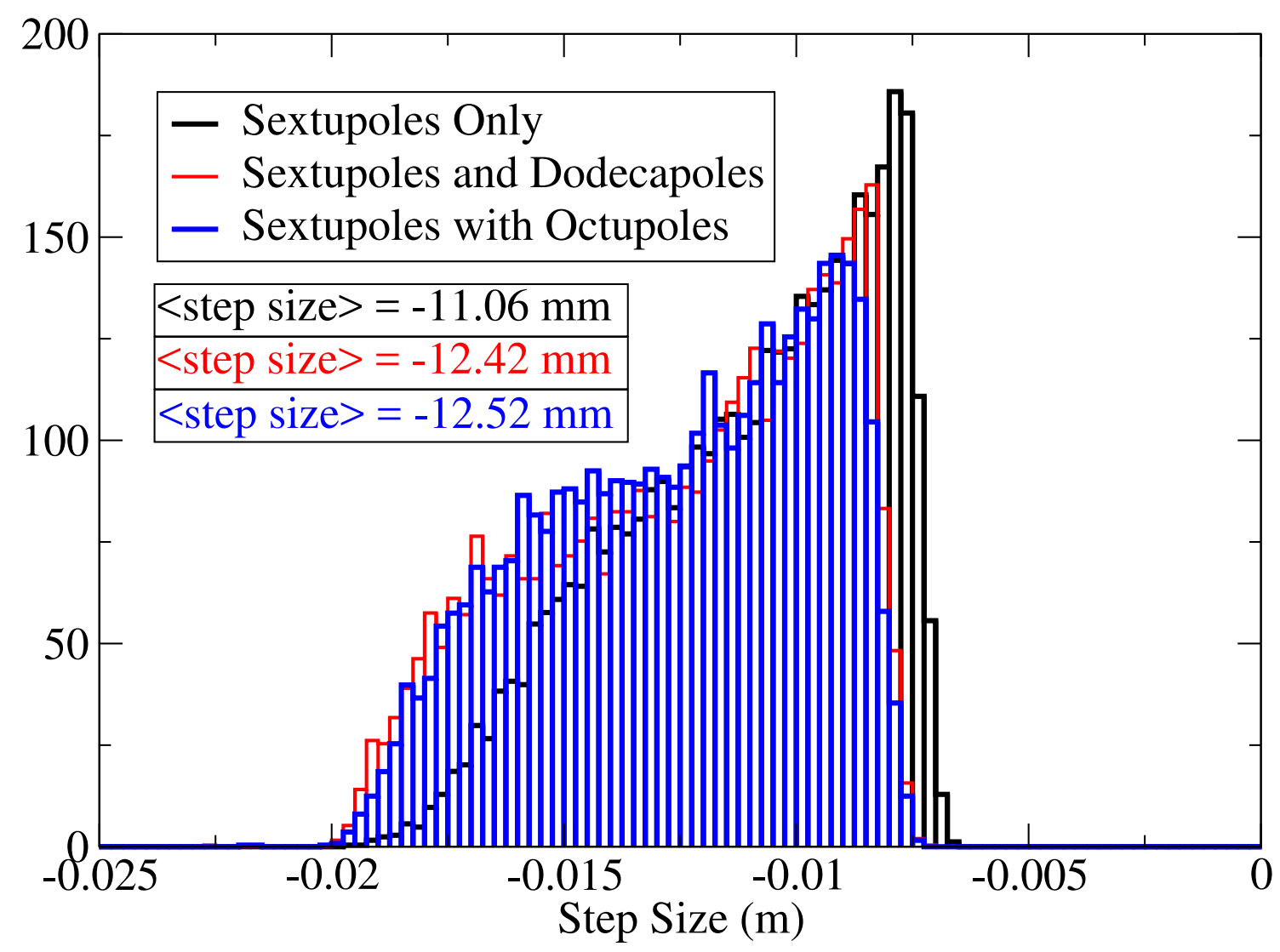

FIG. 10: Step size distributions for normal J-PARC slow extraction, with and without the higher order multipoles. The average step sizes are given for each case.

\section{CONCLUSIONS}

The addition of higher order multiples to the $1 / 3$ integer slow extraction system at JPARC can improve the extraction efficiency and reduce activation on the accelerator components. The higher order multipoles are useful because they bend particle trajectories back inward, allowing the step size to be increased while filling the same amount of aperture.

\section{ACKNOWLEDGEMENTS}

We are very grateful for productive discussions with Dr. Todd Satogata, J.W. Glenn III, and Dr. Ernest Courant. This work was performed under Contract Number DE-AC0298CH10886 with the auspices of the US Department of Energy and with support from the Japanese High Energy Accelerator Research Organization (KEK). 
* Electronic address: kbrown@bnl.gov

$\dagger$ Electronic address: tomizawa.masahito@kek.jp

[1] Y. Yamazaki et al., "Accelerator technical design report for J-PARC," KEK-REPORT-200213, March 2003

[2] M. Tomizawa, Y. Arakaki, S. Machida, Y. Mori, N. Tokuda, T. Yokoi and S. Shibuya, "Design Of Small-Beam Loss Slow Extraction In A High Intensity 50-Gev Proton Synchrotron," Prepared for the 7th European Particle Accelerator Conference (EPAC 2000), Vienna, Austria, 26-30 Jun 2000

[3] J.L. Tuck and L.C. Teng, "Regenerative Deflector for Synchrocyclotron", Phys. Rev. 81, 305 (1951)

[4] A.V. Crewe and K.J. Le Couteur, "Extracted Proton Beam of the Liverpool 156-Inch Cyclotron", Rev. Sci. Instrum. 26, 725 (1955)

[5] K.J. Le Couteur, "The Regenerative Deflector for Synchro-cyclotrons", Proc. Phys. Soc. (London), B64, 1073-1084 (1951)

[6] K.J. Le Couteur, "Perturbations in the Magnetic Deflector for Synchro-Cyclotrons", Proc. Phys. Soc. (London), B66, 25-32 (1953)

[7] H.G. Hereward, "The Possibility of Resonant Extraction from the CPS", CERN AR/Int. GS/61-5 (1961)

[8] H.G. Hereward, "The CPS Resonant Extraction System" Proc. Int. Conf. on High Energy Accel., Dubna (1963)

[9] M.Q. Barton, "Beam Extraction from the Proton Synchrotron", IEEE Transactions on Nuclear Science, pp.660-667, June 1967

[10] Y. Kobayashi and H. Takahashi, "Improvement of the Emittance in the Resonant Beam Ejection", Proc. VIth Int. Conf. on High Energy Accel., Massachusetts, pp.347-351 (1967)

[11] C. Bovet, G.R. Lambertson, and K.H.Reich, "Measurements on Slow Beam-Ejection from the CPS", CERN Report 64-25, 1964

[12] R. Nagaoka, K. Yoshida and M. Hara, "Nonlinear dynamics with sextupoles in low-emittance light source storage rings", Nucl. Instr. and Meth. A 302 (1991), p. 9.

[13] T. Satogata, "Nonlinear resonance islands and modulational effects in a proton synchrotron", 
PhD thesis, Northwestern University (1993)

[14] N.N. Alexeev, S.L. Bereznitsky, A.E. Bolshakov, "Perturbation of Relevant Resonance for Slow Extraction Efficiency Increase", Proceedings of the 1997 Particle Accelerator Conference, Vancouver, B.C., pp. 261-263 (1997)

[15] L. Ahrens, K. Brown, J. W. Glenn, T. Roser, N. Tsoupas and W. van Asselt, "AGS resonant extraction with high intensity beams," Prepared for IEEE Particle Accelerator Conference (PAC 99), New York, New York, 29 Mar - 2 Apr 1999

[16] C. D. Moore, J. Firebaugh, P. Lucas and P. Prieto, "Status Of Resonant Extraction From The Fermilab Main Injector," Prepared for IEEE Particle Accelerator Conference (PAC 99), New York, New York, 29 Mar - 2 Apr 1999

[17] C. Steinbach, H. Stucki and M. Thivent, "The new slow extraction system of the CERN PS," Prepared for IEEE Particle Accelerator Conference (PAC 93), Washington, D.C., 17 - 20 May 1993

[18] Y. Baconnier, P. Faugeras, K. H. Kissler, B. De Raad and W. Scandale, "Extraction From The Cern Sps. (Talk)," IEEE Trans. Nucl. Sci. 24, 1434 (1977).

[19] M. Pullia, "Dynamics of slow extraction and its influence on transfer lines design", PhD thesis, Universite' Claude Bernard Lyon 1 (1999)

[20] C. Steinbach, "Beam Optics at Resonant Extraction Septa", Proceedings of the 1992 European Particle Accelerator Conference, Berlin, pp.857-859 (1992)

[21] P. Strolin, "Resonant Extraction from the CERN Intersecting Storage Rings", CERN Report 69-6, March 1969

[22] D.Edwards and M.Syphers, "An Introduction to the Physics of High Energy Accelerators", Wiley Series in Beam Physics, 1993 John Wiley \& Sons, Inc. ISBN 0-471-55163-5

[23] H.Grote, F.C.Iselin, "The MAD Program User's Reference Manual”, CERN/SL/9013(AP)Rev.4, May 26, 1995. 